\title{
Progress of Brain Natriuretic Peptide in Cardiovascular System Cai-Ling REN
}

Gannan Medical University, Ganzhou 341000, Jiangxi Province, China.

rlingz@163.com

Keywords: Brain natriuretic peptide, Cardiovascular, Exercise.

\begin{abstract}
Brain natriuretic peptide (BNP), mainly secreted by the ventricle, has diuretic natriuretic effect, which can relax blood vessels, inhibit aldosterone secretion and renin activity, and participate in the regulation of blood pressure, blood volume and water and salt balance. Improving glomerular filtration rate, natriuretic diuretic, dilating blood vessels, reducing systemic vascular resistance and plasma volume all play a role in the maintenance of cardiac function.
\end{abstract}

Brain natriuretic peptide (BNP) is a member of the natriuretic peptide family. It was discovered by Sudoh et al in 1988 in the further study of the related compounds of porcine brain natriuretic peptide. [1. BNP is homologous to ANP, mainly secreted by ventricle, and has diuretic natriuretic effect. BNP can relax blood vessels, inhibit aldosterone secretion and renin activity. Synthesis and secretion, physiological action, interaction with other neurohormones and their application in cardiovascular diseases are reviewed.

\section{Biological Characteristics of BNP}

Pig and dog brains have high levels of BNP immune activity, concentrated in medulla, but in humans and monkeys. BNP could be detected in human peripheral blood and heart, and the concentration of BNP in anterior ventricular vein was significantly higher than that in aortic root. Further studies showed that the activity of BNPmRNA in the ventricle was significantly higher than that in the atrium and the activity of ANPmRNA was higher than that in the atrium, while the content of ANP in the ventricle of the patients with heart failure was higher than that in the patients with heart failure. However, compared with the atrium, it is still in the secondary position, indicating that the synthetic and secretory sites of BNP and ANP are different, and the ventricle is BNP. The concentration of BNP in human plasma can be measured by radioimmunoassay, and the level of BNP in normal human plasma varies with the laboratory.

\section{BNP and Cardiovascular Disease}

\section{Cardiovascular Effects of BNP}

BNP and ANP are both natural antagonists of renin-angiotensin-aldosterone system, and also resist vasopressin and sodium preservation of sympathetic nerve. Maintenance of blood pressure. BNP, together with ANP, is involved in regulating blood pressure, blood volume, water and salt balance, increasing glomerular filtration rate, natriuretic diuretic, and vasodilating. Decrease of systemic vascular resistance and plasma volume, which can maintain cardiac function. BNP is different from ANPANP mainly in atrial synthesis and secretion is increased during atrial overload or dilation. Increased plasma concentration, major adverse effects Reflecting changes in pulmonary vascular pressure, other hormones such as antidiuretic hormones, catecholamines can directly stimulate the secretion of ANP because ANP precursors are stored in secretory granules. When secreting, it is decomposed into ANPs, and its rapid regulation is mainly carried out on the amount of hormone secretion. However, BNP is mainly synthesized in the ventricle and increased during ventricular overload or dilation. Therefore, it is more sensitive and specific to reflect the changes of ventricular function, because the precursor of BNP is not stored in secretory granules. The rapid regulation of BNP synthesis and secretion takes place at the level of gene expression. 
Heart failure is the end stage of various diseases, heart failure can be divided into acute congestive heart failure (CHF), compensatory left ventricular dysfunction CLVD) / asymptomatic left ventricular dysfunction (AL CD), symptomatic left ventricular dysfunction (SL VD) / obvious symptoms of heart failure (OHF) and other types of heart failure, hemodynamic change, systemic and local hormones involved in myocardial and renal vascular changes, the adaptability of the natriuretic peptide system was also significantly activated, the plasma BNP level of heart failure severity increased significantly. BNP has a close relationship with heart function based on many researchers made great The quantity of work to explore its clinical application.

\section{BNP and Left Ventricular Dysfunction, Congestive Failure}

The data of the population over the age of 45 , men with chronic congestive heart failure (CHF) the incidence rate of /1000 in 72 cases, female 47 cases of /1000 patients with heart failure, the average survival time of 17 years for males and 32 years for women, so the heart failure is quite common, and the prognosis is poor. However, it is difficult for clinical diagnosis and differential the diagnosis of heart failure before, mainly depends on the ECG, X and echocardiography, the lack of sensitive and specific laboratory indicators, the misdiagnosis rate is high, especially for mild and moderate heart failure patients. At present, many scholars have suggested that the BNP for CHF In the diagnosis of CHF and left ventricular dysfunction, BNP was compared with echocardiographic diagnosis. It was found that $51 \%$ of the patients (no history of CHF and left ventricular dysfunction) had a BNP concentration of 328pg / $\mathrm{mL}$. The specificity was significantly higher than that in the normal control group (30 PG / mL), and the specificity was $95.8 \%$. But it was found in 72 patients with symptomatic left ventricular dysfunction who had increased systolic diastolic ventricular dysfunction. Maeda et al. Plasma BNP concentration and left ventricular end-diastolic pressure measured by cardiac catheterization After treatment with angiotensin-converting enzyme inhibitor (ACEI), both of them decreased, showing a high correlation. Therefore, BNP is a noninvasive blood biochemical marker for evaluating ventricular dysfunction. Cowie et al., compared with clinical diagnosis, compared BNP with clinical diagnosis. The results showed that the area under the ROC curve of BNP was 0.98, indicating that the accuracy of BNP in the diagnosis of CHF was very high. The accuracy of clinical diagnosis of CHF is only 29. Therefore, plasma BNP can be used as a screening method for ventricular dysfunction. According to Alan S. [24] data display Plasma BNP concentrations in patients with heart failure, left ventricular dysfunction and non-heart failure were $675 \pm 450 \mathrm{ng} / \mathrm{L}, 346 \pm 390 \mathrm{ng} / \mathrm{L}$ and $110 \pm 225$ PG / mL, respectively. The results showed significant difference. If cardiac insufficiency was classified into four grades according to the criteria of the New York Heart Association, the concentration of BNP in grade I $\sim$ IV was 244 \pm 286 PG / mL. $389 \pm 374 \mathrm{pg} / \mathrm{mL} 640 \pm 477 \mathrm{pg} / \mathrm{mL}$ and $817 \pm 435 \mathrm{pg} / \mathrm{mL}$. if $100 \mathrm{pg} / \mathrm{mL}$ is the critical value. Sensitivity and sensitivity in the diagnosis of heart failure The specificity was $90 \%$ and 76 respectively. In order to explore the relationship between the concentration of brain natriuretic peptide (BNP) and the severity of cardiac insufficiency and left ventricular systolic function, it was found that the concentration of BNP in the observation group was significantly higher than that in the control group. The concentration of BNP was positively correlated with the grading of NYHA. There was a good correlation between the concentration of BNP and the degree of left ventricular systolic insufficiency, which indicated that the concentration of BNP could reflect the severity of heart failure and the degree of left ventricular systolic insufficiency.

\section{BNP and Atherosclerosis}

ACS is a group of myocardial ischemic diseases, classified by severity, from unstable angina pectoris to acute myocardial infarction (AMI). Its common pathophysiological characteristics are alternating coronary spasm, atherosclerotic plaque fall off. Platelet aggregation and thrombus formation. Recently, it was found that BNP was closely related to AMI and prognosis. Plasma BNP increased rapidly within 24 hours after the onset of AMI, and then tended to a relatively stable value. Plasma BNP concentration was closely correlated with left ventricular ejection fraction (LVEF) in patients with transmural AMI within 4 days. In the near term and in the medium term, BNP was associated with the prognosis. It is very significant that.Omland BNP than other common risk 
factors is more valuable, the higher the BNP, the long-term prognosis is very poor. Many studies showed that plasma levels of BNP and AMI in the infarction area, ischemic area and UPI was positively associated with left ventricular ejection fraction and cardiac index were negatively correlated. BNP as the efficacy index, reduce AMI mortality and disability rate. The increase of plasma concentrations of BNP can provide important physiological characteristics in patients with ACS. Three kinds of warning information has the potential to resist chemotactic natriuretic peptide on vascular smooth muscle cell proliferation. The effect. Casco et al. The presence and distribution of natriuretic peptide and receptors in human coronary atherosclerosis were detected by cell immunocytochemistry and in situ hybridization. It was found that there were different manifestations in different degrees of atherosclerosis. In the early lipid striation stage, only the low level of ANP can be detected, and in the middle stage, a large amount of ANP can be detected. CNP and their receptors have more BNP when the atherosclerosis is high, which can be used as a predictor of the high risk of ACS. Therefore, for people at risk of ACS The BNP level should be detected whether or not there are St segment changes or other biochemical changes. If there is a high level of BNP, attention should be paid to it.

\section{BNP and Hypertension}

Left ventricular hypertrophy is an important complication of essential hypertension. Nishigaki et al observed that the plasma BNP concentration in hypertension was significantly higher than that in patients with critical hypertension and normal blood pressure. It was also correlated with the degree of blood pressure progression. Left ventricular hypertrophy was significantly higher in patients with left ventricular hypertrophy than in those without left ventricular hypertrophy, and had a close positive correlation with the left ventricular mass index. The level of BNP in hypertensive patients with left ventricular hypertrophy $(\mathrm{LVH})$ was significantly decreased after ACEI treatment from June to December, and the left ventricular mass index (LVMI), septum and posterior wall thickness were significantly decreased. Determination of plasma BNP level may be used as a predictor of essential hypertension after antihypertensive therapy. Whether left ventricular hypertrophy can be reversed. The increase of BNP concentration in essential hypertension may be a compensatory factor. However, the exact mechanism remains to be further studied. It may be due to ventricular wall tension and increased vascular wall brittleness to stimulate the synthesis and secretion of BNP. Therefore, early detection of BNP in these populations. Hypertension and left ventricular hypertrophy can be detected and diagnosed early.

\section{BNP and Exercise}

A study was conducted on 8 healthy men with the same target center rate and the same exercise time. Results: plasma atrial natriuretic peptide (ANP) and brain natriuretic peptide (BNP) were significantly increased after isometric exercise, but not after anti exercise. However, the degree of secretion is positively correlated with the two products, suggesting that exercise can lead to the increase of plasma natriuretic peptide hormone, and there is a phenomenon of interaction between the levels of each hormone in the body. Both types of exercise can improve the storage function of coronary vessels.

\section{Research Prospect}

Due to the synthesis, secretion, physiological and pathophysiological characteristics of BNP, it can be used as an index to judge the ventricular function of patients with left ventricular function, acute respiratory failure and hemodialysis after the recovery of heart disease. It can also be used as a biochemical index of ventricular remodeling within 30 days of acute myocardial infarction to determine the risk of sudden death in asymptomatic patients with heart failure and after myocardial infarction. N- terminal BNP was used as a screening method for left ventricular systolic disorder in normal population.. The study of natriuretic peptides is very active at present. The basic profile of cardiovascular homeostasis is clear, but there are still many problems. The comprehensive study of 
$\mathrm{P}$ is on the rise and is in the ascendant.

\section{References}

[1]Kemperman H, Vand BM, Kirkels H, et al. B-type natriuretic peptide(BNP) and N-terminal proBNP in patients with end-stage heart failuresupported by a left ventricular assist device. Clinical Chemistry, 2004, 50:1670-1672.

[2]Maisel A K ,Koon J Hope $\mathrm{J}$,et al .A rapid beside test for brain natriuretic peptide accurately predicts echocardiogmphy [J] .Am Heart J ,2001,141; 374-379

[3]Maeda K , Tsutamoto T Wada A, et al .Plasma brain natriuretic peptide as a biochemical marker of high left ventricular end-diastolic pressure in patients with symptomatic left ventricular dysfunction [J],1998,135(5 Pt1):825- 832.

[4]Cowie M R , Struthers AD ,Wood DA ,et al. Value of natriuretic peptides in assessment of patients with possible new heart failure in primary care[J] .Lancet ,1997,350(9088);1349-1353

[5]Alan S ,Padma $\mathrm{K}$,Richard $\mathrm{M}$,et al .Rapid measurement of B-type natriuretic peptide in the emergency diagnosis of heart failkure [J] . N Eng J Med , 2002,347:161-167

[6]Tjeerdsma $\mathrm{G}$, de boer RA , Boomsna $\mathrm{F}$, et al .Rapid beside measurement of brain natriureetic peptide in patients with chronic heart failure[J]. Int J Cardiol , 2002 , 86(2-3) ; 143-149

[7]Troughton R W ,Frampton C M ,Yandle T G ,et al ,Traetment of heart failure guided by plasma a minoter minal brain natriuretic peptide (N-BNP) concentrations [J]. Lancet , 2002,106(23);2913-2918

[8]Omland $\mathrm{T}$,Person A , Ng L ,O’Brien R ,et al .Nterminal pro-B-type natriuretic peptide and long-term mortality in acute coronary syndromes[J]. Circulation ,2002 , 108(23); 2923-2915

[9]Casco V, Veinot J P ,Kuroski de Bold M L ,et al .Natriuretic peptide system gene expression human coronary arteries [J] . J Histochbem Cytochem ,2002,50(6);799-809

[10]Nishigaki K ,Tomita $\mathrm{M}$,Kagawa $\mathrm{K}$,et al .Marked expression of plasma brain natriuretic is a special feature of hypertophic obstructive cardiomyopathy[J]. J Am Coll cardiol ,1996,28;123-142

[11]J Keda T , Matsuda $\mathrm{K}$,Itoh $\mathrm{H}$, et al .Plasma levels of brain and atrial natriurtic peptides elevate in proportion to left ventricular wall stress in patients with aortic stenosis[J] ,Am Heart J , 1997,133;307-314 